\title{
Power Dependent of Nonlinear Absorption Coefficient of CdSe-Au Hybrid Nanocomposites
}

\author{
Abeer Salah, Mona Bakr, Salah Hassabelnaby, Iftitan Azzouz, Yehia Badr \\ National Institute of Laser Enhanced Sciences, Cairo University, Giza, Egypt \\ Email: abeersalah2014@gmail.com \\ Received 26 February 2015; accepted 12 April 2015; published 15 April 2015 \\ Copyright (c) 2015 by authors and Scientific Research Publishing Inc. \\ This work is licensed under the Creative Commons Attribution International License (CC BY). \\ http://creativecommons.org/licenses/by/4.0/

(c) (i) Open Access

\begin{abstract}
Power dependent study of optical nonlinear absorption coefficient of semiconductor-plasmonic hybrid nanocomposites of CdSe:Au was investigated using Z-scan technique, provided with nanosecond pulses of second harmonic Nd:YAG laser. The prepared materials were characterized with linear absorption and High Resolution Transmission Electron Microscope (HRTEM) which confirm the quantum confinement of CdSe as well as the formation of CdSe:Au core shell quantum dots. An analysis of power dependence open aperture Z-scan was discussed. A change from saturable absorption (SA) to reverse saturable absorption (RSA) is observed with increasing the laser power. Also, an enhancement of the nonlinear absorption coefficient of CdSe:Au of larger sizes compared to Au nanoparticles was detected.
\end{abstract}

Keywords

Z-Scan, Nonlinear Absorption, Optical Limiting, CdSe:Au

\section{Introduction}

Nanoparticles have enormous applications in photonic devices, such as, optical limiter [1] [2] and all optical switching [3] [4].

Optical limiters are those materials which limit intense laser beam to a low level. They exhibit large nonlinear extinction response at higher intensities but permit higher transmittance at low intensities [5]. One or more nonlinear optical processes like free carrier absorption, multi-photon absorption and nonlinear scattering were occurred as the origin of their optical limiting performance [1] [2] [6]. Optical limiters have enormous applications such as eye protection, sensors and optical imaging devices can be achieved by keeping transmitted energy 
below the damage threshold [5].

The properties of semiconductor nanoparticles result from further confinement of the electronic motion to a length scale that is comparable to or smaller than that characterizing the electronic motion in bulk semiconducting material (called the electron Bohr radius, which is usually a few nanometers) [7]. The properties of metallic nanoparticles result from the collective oscillation of the electrons in the conduction band from one surface of the particle to the other. This oscillation has a frequency that absorbs the visible light. This is called the surface plasmon resonance.

The unique properties of the metal and semiconductors can combine and also can generate collective new phenomena based on the intraparticles interaction between the plasmonic effect of the metal core and the semiconductor shell at their interface when a metal and a semiconductor in the same nanocrystals [8].

Z-scan measured the transmittance of a nonlinear medium, detected through a finite aperture in the far field as a function of the sample position $(\mathrm{Z})$ with respect to the focal plane. The open aperture transmittance (the aperture in front of the detector was removed) used to measure the nonlinear absorption coefficient, while the closed Z-scan can be used to measure the nonlinear refractive index. The most important advantage of Z-scan is an indication of the sign and magnitude of nonlinearity.

The nonlinear absorption NLA can be either saturable absorption (SA), where the absorption coefficient decreases with increasing intensity, or reverse saturable absorption (RSA), where the absorption coefficient increases with increasing intensity. RSA can occur when the absorption cross-section of excited states exceeds the ground state cross section but for SA the cross-section of excited state less than the ground state cross section. At the focal point, the sample experiences maximum pump intensity, which gradually decreases in either direction from the focused beam. Saturable and reverse saturable absorption are the phenomena's studies in the open aperture Z Scan in the form of increase (peak) or decrease (valley) of the transmittance curve at the focus spot

The normalized transmittance can be expressed as [9]:

$$
T_{\text {Norm }}(z)=\sum_{m=0}^{\infty}\left(\frac{-\beta I_{0} L_{e f f}}{1+z^{2} / z_{o}^{2}}\right) /(m+1)^{3 / 2}
$$

where $q_{0}=\beta I_{0} L_{\text {eff }}, \quad \beta$ is the nonlinear absorption coefficient, $L_{\text {eff }}=\frac{1-\mathrm{e}^{-\alpha_{o} L}}{\alpha_{o}}$ is the effective interaction length with the sample thickness $\mathrm{L}$, and $\alpha_{o}$ is the linear absorption coefficient, Thus $\beta$ can be determined by fitting this expression to the experimental results.

We can obtain $\operatorname{Im} \chi^{(3)}$ in terms of the following relation [9]

$$
\operatorname{Im} \chi^{(3)}=\frac{n_{0}^{2} \varepsilon_{0} c^{2}}{4 \pi^{2}} \lambda \beta
$$

As the proximity of the exciton energy of CdSe and the surface plasmon resonance excitation energy for $\mathrm{Au}$ nanoparticles offers the opportunity to produce nanocomposite materials in which excitation of the CdSe leads to rapid charge injection into the Au. We measured the nonlinear absorption coefficient of CdSe:Au as a function of particle size as well as concentration elsewhere [10]. The importance of doing open Z-scan experiment at different intensities is to reveal possible competing and accompanying process, which may influence the type of the nonlinear absorption. Therefore in this work, Power dependent open aperture Z-scan technique has been used to measure the nonlinear absorption of CdSe:Au hybrid composite of different particle size. Comparison of the nonlinear absorption coefficients of CdSe:Au with Au seed also discussed.

\section{Experimental Techniques}

Gold nanoclusters have been prepared via pyrolysis of organometallic [11] by injection of Au: $\mathrm{HCl}(10 \mu \mathrm{L})$ solution into a mixture of oleic acid and alkyl amine such as oleyl amine (OA) or hexadecyl amine (HAD ) in microwave for 30 seconds. The solution color turned to be finite pink. This is an indication of the formation of small clusters of gold sample, which have been confirmed by the absorption spectra and the TEM images. This solution of gold particles was used as a seed (Au seed). Preparation details of CdSe:Au had been described in ref [12].

The HRTEM images were taken with JEM JEOL-2010 transmission electron microscope with an accelerating 
voltage of $200 \mathrm{kV}$. Solution of methanol has been added to the samples to separate from the reaction mixture and the bi-product. The solution was centrifuged, and then the precipitated was re-dispersed in toluene.

The linear absorption properties of the composites were investigated using Perkin Elmer Lambda 35 spectrometer in the range from $400-800 \mathrm{~nm}$. The linear absorption coefficient $\alpha_{0}$ at $532 \mathrm{~nm}$ is a prerequisite for measuring the nonlinear absorption coefficient. It is determined in an easy way using beer's law, which operates in the linear absorption regime.

The signs and values of nonlinear absorption coefficient were determined by open aperture Z-scan technique. a Q-switched $532 \mathrm{~nm}$ frequency doubled Nd: YAG laser (Continuum) characterized by pulse duration of $6 \mathrm{~ns}$ was employed. The pulse repetition rate was set at $1 \mathrm{~Hz}$ to reduce the possible thermal accumulative effect. The laser energy was varied from $50 \mu \mathrm{J}$ to $1500 \mu \mathrm{J}$ using different neutral density filters. These energy values were below the optical breakdown threshold for the investigated samples.

The sample moved along the propagation direction of the focused beam by translation through an automatic integrated long travel stage of $150 \mathrm{~mm}$ length (LTS 150 M THORLABS). The focal length of the lens was 150 $\mathrm{mm}$. The radius of the beam waist $\left(\omega_{0}\right)$ was calculated to be $58 \mu \mathrm{m}$.

In order to reduce the influence of the laser power fluctuation, the transmitted beam energy, the reference beam energy and their ratio were measured by a dual channel optical power meter (PM 320 E Thorlabs) with two photodiode detectors (S142C Thorlabs) and the on axis intensity at the focus were $1.85 \times 10^{12}$ to $2.36 \times 10^{13}$ $\mathrm{W} / \mathrm{m}^{2}$.

Figure 1 describes the experimental setup of open aperture $\mathrm{Z}$ scan. The absorptive optical limiting measurements were performed by placing the sample in a quartz cuvette with thickness of $3 \mathrm{~mm}$. The samples were placed at the focal point of the lens.

\section{Results and Discussions}

The linear absorption spectra of Au nanoparticles have a surface Plasmon resonance at $528 \mathrm{~nm}$, which is attributed to the oscillation of free electrons in the conduction band. Figure 2 shows the linear absorption spectrum of Au nanoparticles (Au seed) and different sizes of CdSe:Au. The absorption peaks are 538, 576, 590, 600 and $613 \mathrm{~nm}$ corresponding to average quantum dots sizes of 3.37, 3.54, 3.91, 4.56 and $5.07 \mathrm{~nm}$, respectively, The absorption spectrum shows the excitonic features which are attributed to absorption by CdSe and the surface plasmon resonance (SPR) of Au nanoparticles, it can be deduced that the absorption maximum is red shifted as the particle size increased, while Gerdova et al. [13] showed that the linear absorption of CdSe core quantum dots in toluene with a mean diameter of $2.4 \mathrm{~nm}$ is at $2.24 \mathrm{eV}$ (i.e. absorption band (551 nm)) and Zhang et al. [14] prepared the colloidal Au-CdSe core shell with absorption band approximately at $2.1 \mathrm{eV}$ i.e. $(590 \mathrm{~nm})$.

HRTEM is employed to determine the average particle size of quantum dots QDs as well as to confirm the existence of core-shell particles. Figure 3 shows HRTEM of CdSe:Au of different sizes. The Au particles appear as a dark sphere. These images clearly illustrate the mono-dispersity of CdSe QDs that have a diameter of $3.37-5.07 \mathrm{~nm}$ at different time interval from the preparation, while the size of Au particles in the composite is 8.36 - $10 \mathrm{~nm}$. Since the size of CdSe QDs is smaller than the exciton Bohr diameter of CdSe $11.2 \mathrm{~nm}$, a strong quantum confinement effect should be expected.

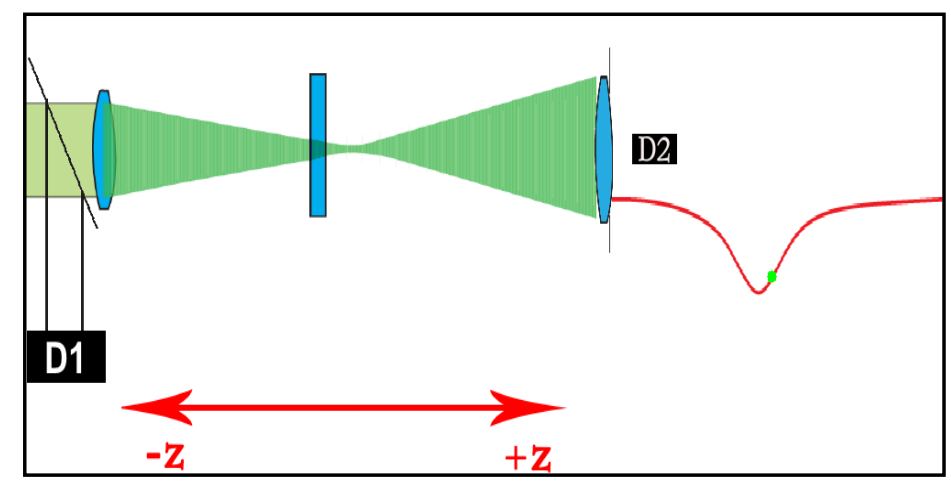

Figure 1. Open Aperture $\mathrm{Z}$ scan, illustraing the position the sample around the focus, the resultant $Z$ Scan curve. 


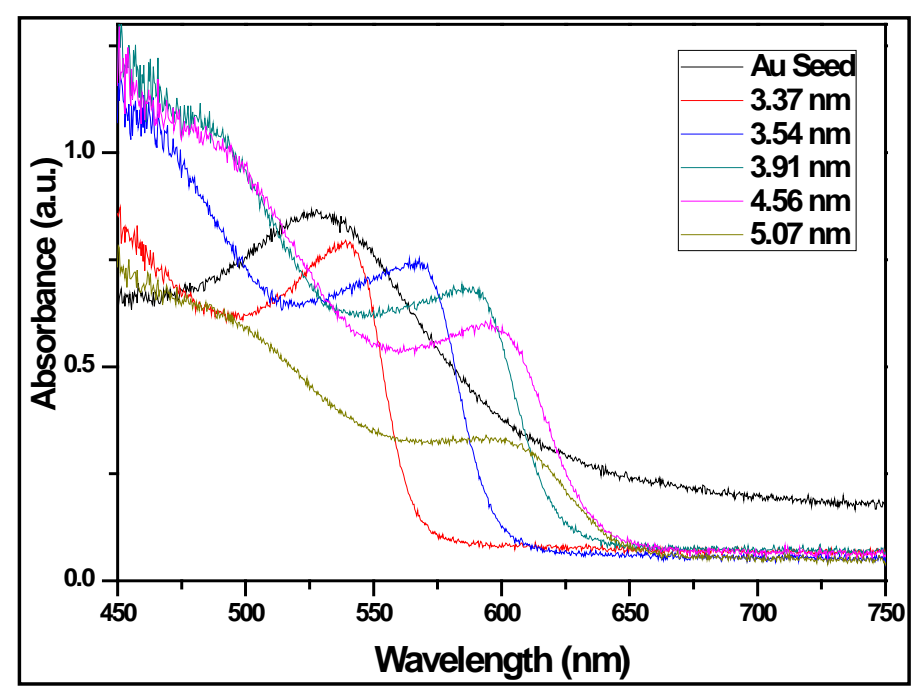

Figure 2. The Linear absorption spectra of Au seed and CdSe:Au hybrid composites of different sizes.
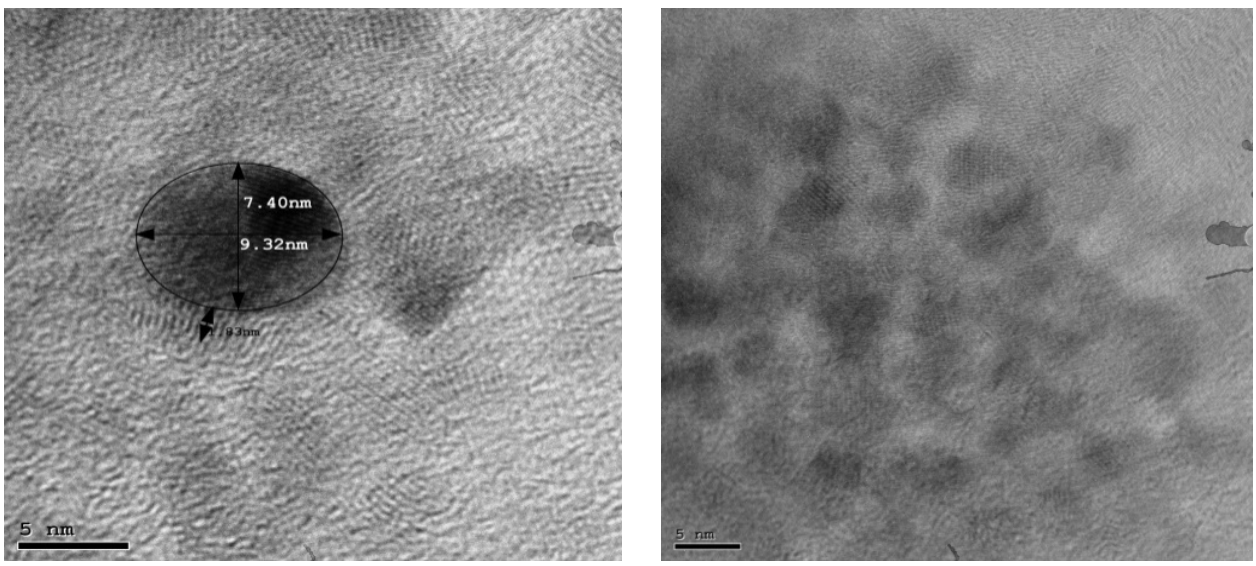

Figure 3. HRTEM of CdSe:Au Hybrid compositesof different sizes. Confirmation of the existence of Au core.

Electron diffraction pattern confirms the existence of gold nanoparticles capped with CdSe and the quantum dot CdSe. In addition, d-spacing can be determined from the diffraction pattern (Figure 4). The d-spacing of $2.31 \AA$ and $1.34 \AA$ correspond to the (111) and (220) planes, respectively, of Au.

The nonlinear absorption of the Au seed (oleic acid + olyelamine) was studied at different powers using open aperture Z scan technique. Open Aperture Z-Scan at different energies for Au (seed) is shown in Figure 5. Open aperture Z scans showed a competition between saturable absorption (SA) and reverse saturable absorption (RSA) of $\mathrm{Au}$ at lower intensities but as the intensity increases the reverse saturable absorption increases and become dominant at $1496 \mu \mathrm{J}$.

Dependance of the nonlinear absorption coefficient on the power intensity for (Au Seed) is shown in Figure 6. the nonlinear absorption coefficient calculated by theoretical fitting to the experimental measurements using Equation (1). The nonlinear absorption coefficient is $-1.1 \times 10^{-10} \mathrm{~m} / \mathrm{W}$ at low intensity and changed to positive value $+3.4 \times 10^{-11} \mathrm{~m} / \mathrm{W}$ at high intensity.

Open aperture Z scan for CdSe:Au at different laser energies is shown in Figure 7, for CdSe:Au at CdSe size $3.37 \mathrm{~nm}$ (a), The normalized transmission exhibits two peaks and a small one valley with respect to the focus $(\mathrm{z}=0)$ at $118 \mu \mathrm{J}$, implying that the SA plays a primary role for the nonlinear properties of material. As the value of the intensity $\mathrm{I}_{0}$ continues to increase the valley in the Z-scan trace is monotonously enhanced, and the peaks are reduced gradually, as shown in Figure 7(a). It can be deduced that the reverse saturable absorption effect 

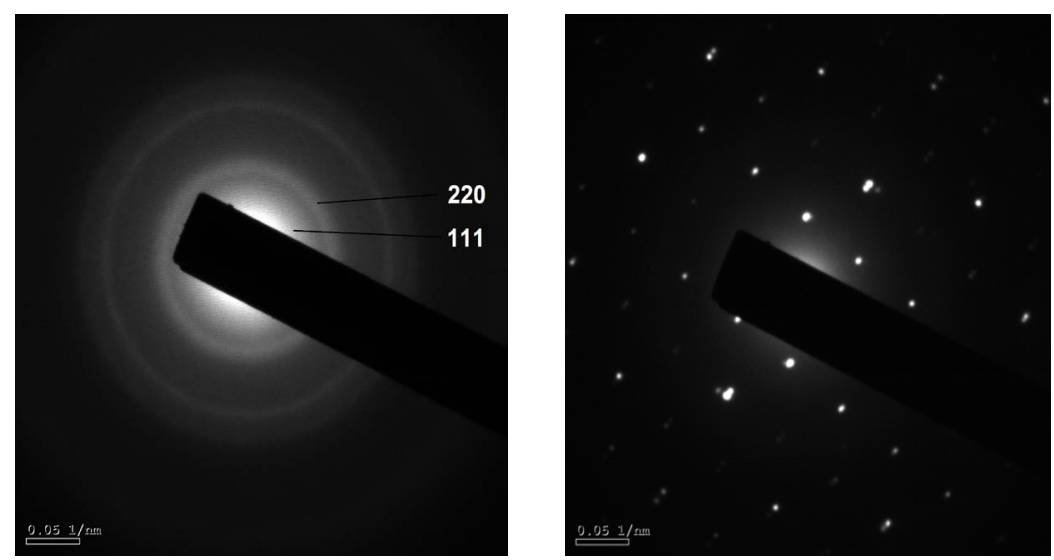

Figure 4. Diffraction pattern of CdSe:Au.

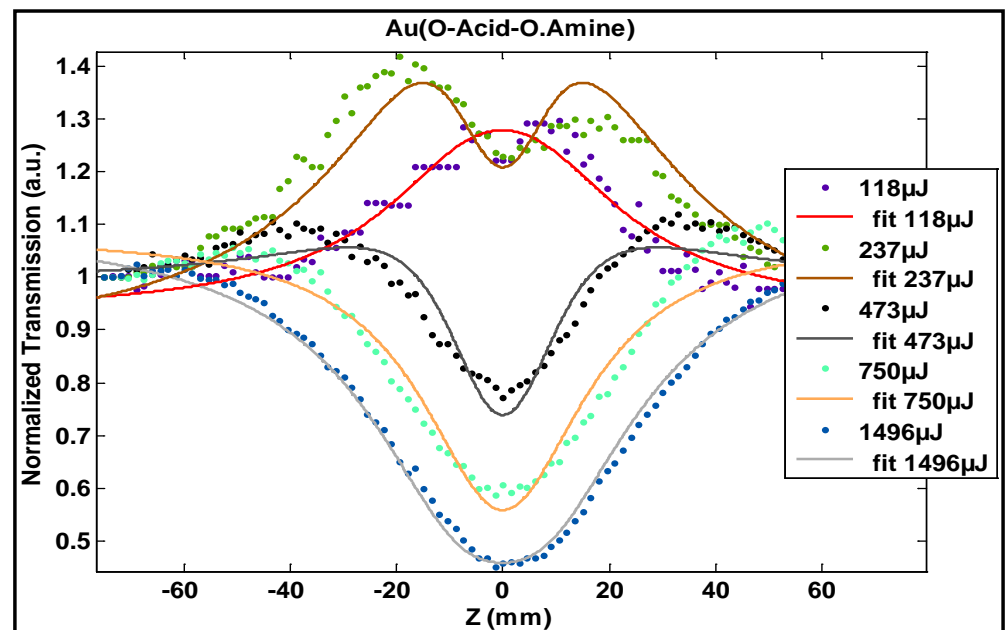

Figure 5. Open Aperture Z-Scan at different energies Au (seed).

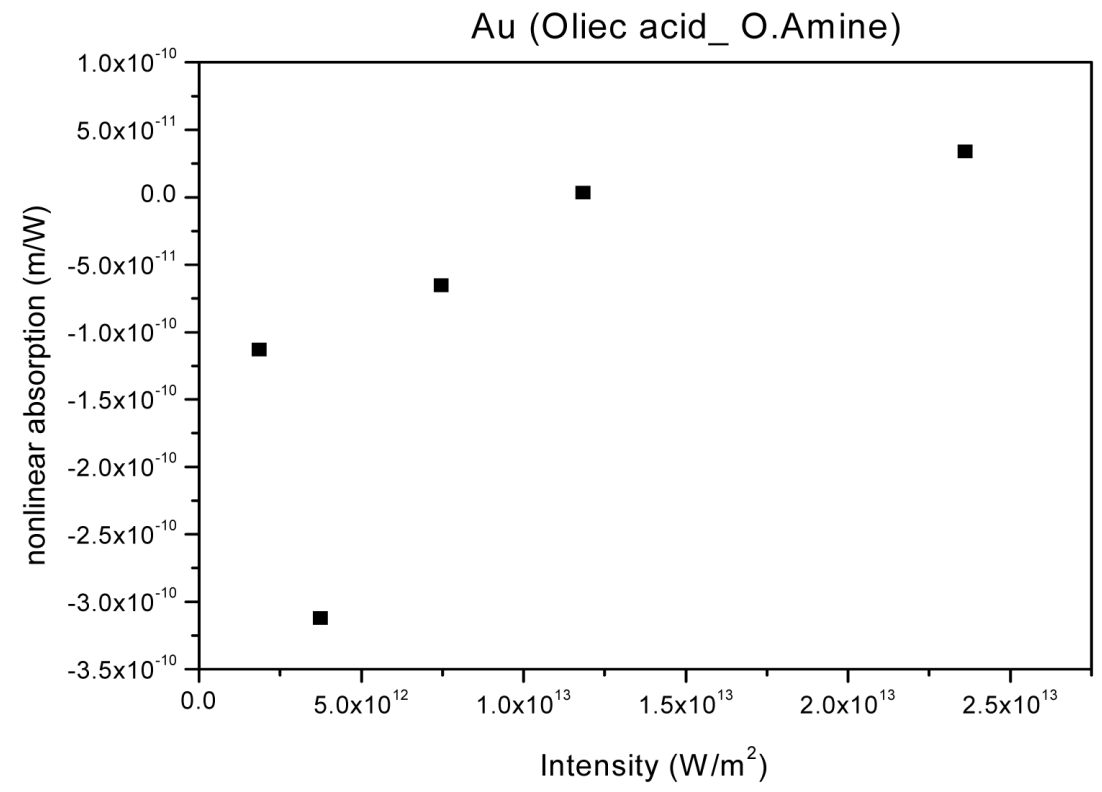

Figure 6. Depenence of the nonlinear absorption coefficient on the power intensity for Au Seed. 


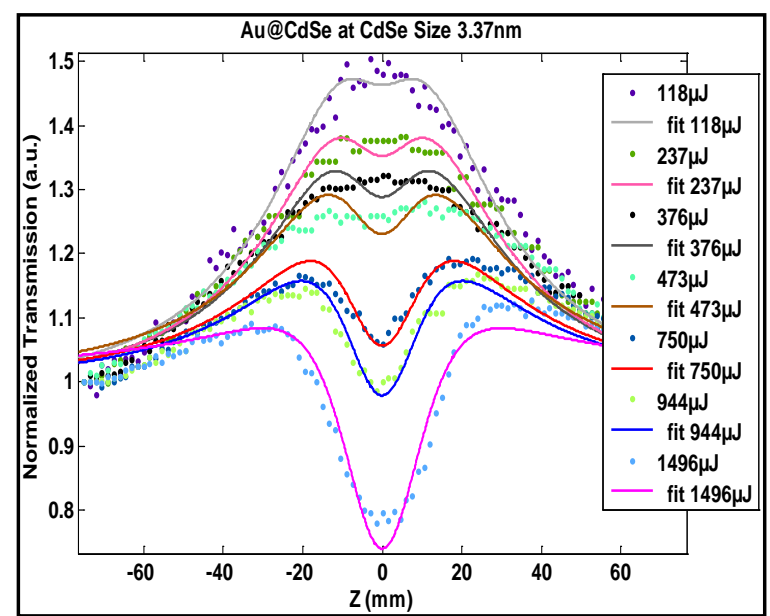

(a)

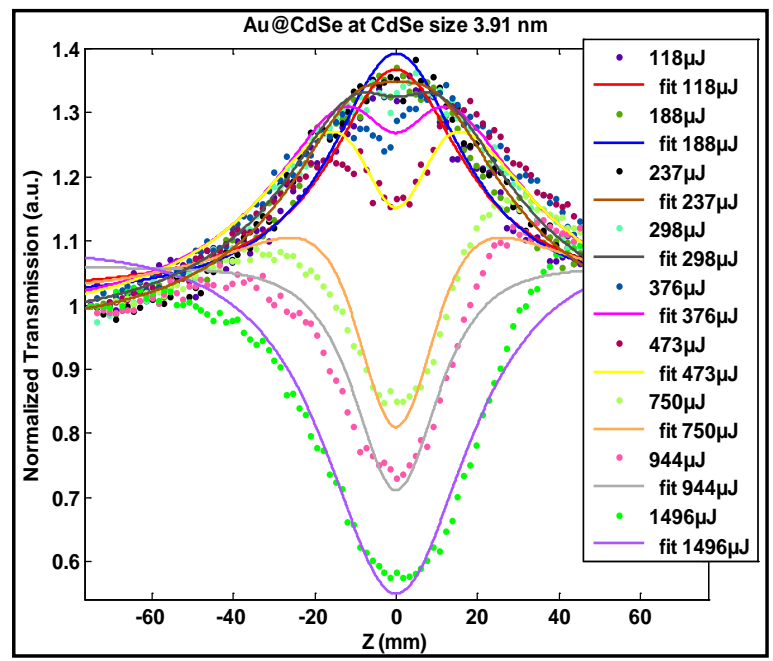

(c)

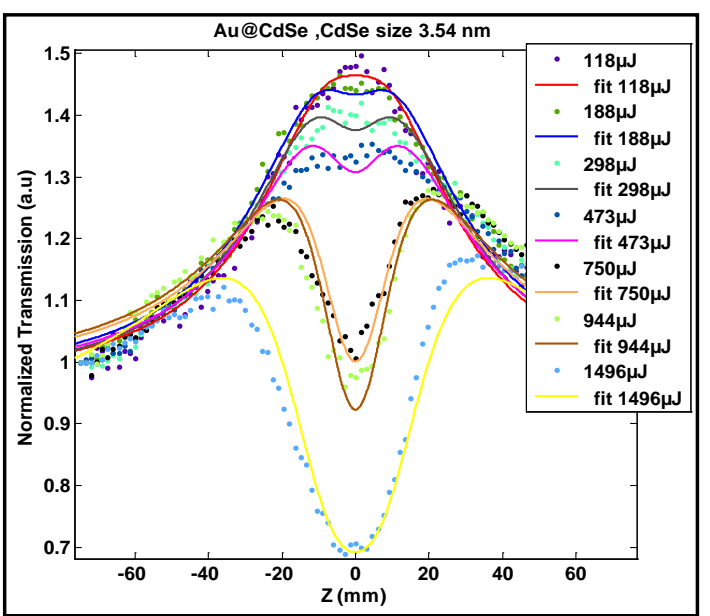

(b)

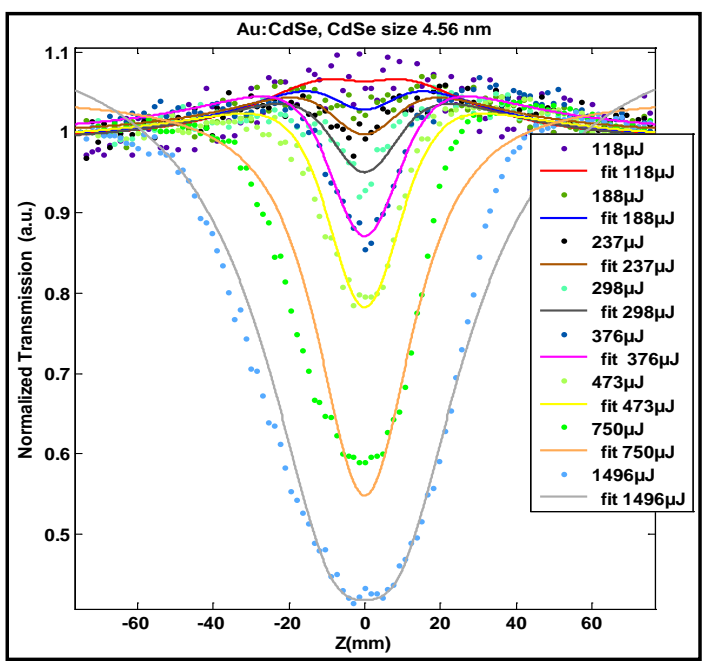

(d)

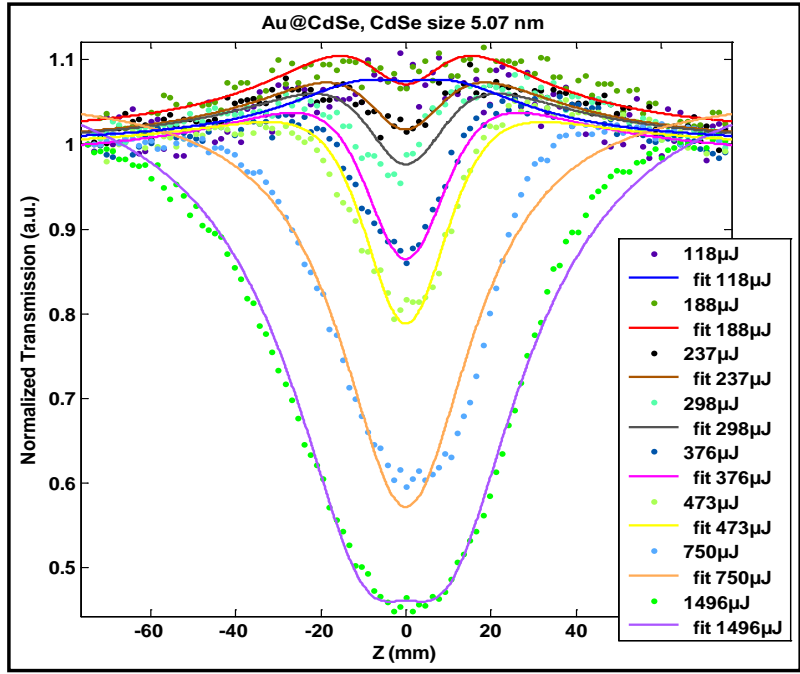

(e)

Figure 7. Open aperture Z-scan at different laser energies for CdSe:Au (a) at CdSe size $3.37 \mathrm{~nm}$; (b) at CdSe size 3.54 $\mathrm{nm}$; (c) at CdSe size $3.91 \mathrm{~nm}$; (d) at CdSe size $4.56 \mathrm{~nm}$; (e) at CdSe size $5.07 \mathrm{~nm}$. 
gives rise to the enhancement of the valley in the Z-scan trace. And, the nonlinear absorption coefficient is affected by the existence of the two competing effects (SA and RSA). So the intensity-dependent transmittance curves (when the sample is located at $\mathrm{z}=0$ ) can be used to identify whether the material possesses simultaneous TPA and SA or not.

It can be noticed that the depth of the valley increase for larger size of CdSe at the same intensity, for example by noticing the figures at $750 \mu \mathrm{J}$ (Figure 7(a) and Figure 7(b)), we have two humps and a valley around the focus. However, the humps are decreased and the valley is increased around the focus as shown in Figure 7(c) and Figure 7(d), finally in Figure 7(e), no humps and completely valley around the focal point at $750 \mu \mathrm{J}$. so it can be deduced that as the size of quantum dots of CdSe increased in CdSe:Au composites, the effect of RSA increased. This might be due to cross section of excited states are greater than the cross section of the ground state for larger particles.

The nonlinear absorption coefficients for CdSe:Au was calculated by theoretical fitting to the measurements using Equation (1). Therefore, dependence of the nonlinear absorption coefficient on the intensity for CdSe:Au is shown in Figure 8. It should be noted that the magnitude of $\beta$ is decreased as the intensity increased due to the presence of the saturable absorption and the competing effect of the reverse saturable absorption. This might be an indication that the cross sections of the excited states become larger than the ground states along the $\mathrm{Z}$ scan.

The dependance of the nonlinear absorption on composite size for CdSe:Au is shown in Figure 9. By analyzing the nonlinear absorption of different composite size (CdSe:Au) at high laser energy (1496 $\mu \mathrm{J})$, the nonlinear absorption coefficient is changed from negative to positive value as the size of composite increased. The saturable absorption switches to RSA with different sizes. As the absorption band of the hybrid composite shifted to a longer wavelength from the excitation line, the RSA is begin to appear at a lower intensity (a lower energy).

Comparing the nonlinear absorption of CdSe:Au hybrid nanocomposites to that of Au (seed), the CdSe QD may be responsible for the SA, as the size of the quantum dot decreased the SA becomes predominant for CdSe:Au. It is clear that as the size of quantum dots in CdSe:Au hybrid composites decreased, the SA appeared to higher intensities, so it can be used for optical limiting at higher intensities and also the larger sizes can be used for optical limiting for lower intensities.

Obviously, the nature of the nonlinear absorption depends on the position of excitation wavelength relative to the SPR (Surface Plasmon Resonance) of the nanomaterial, when the electrons in the ground state are excited by

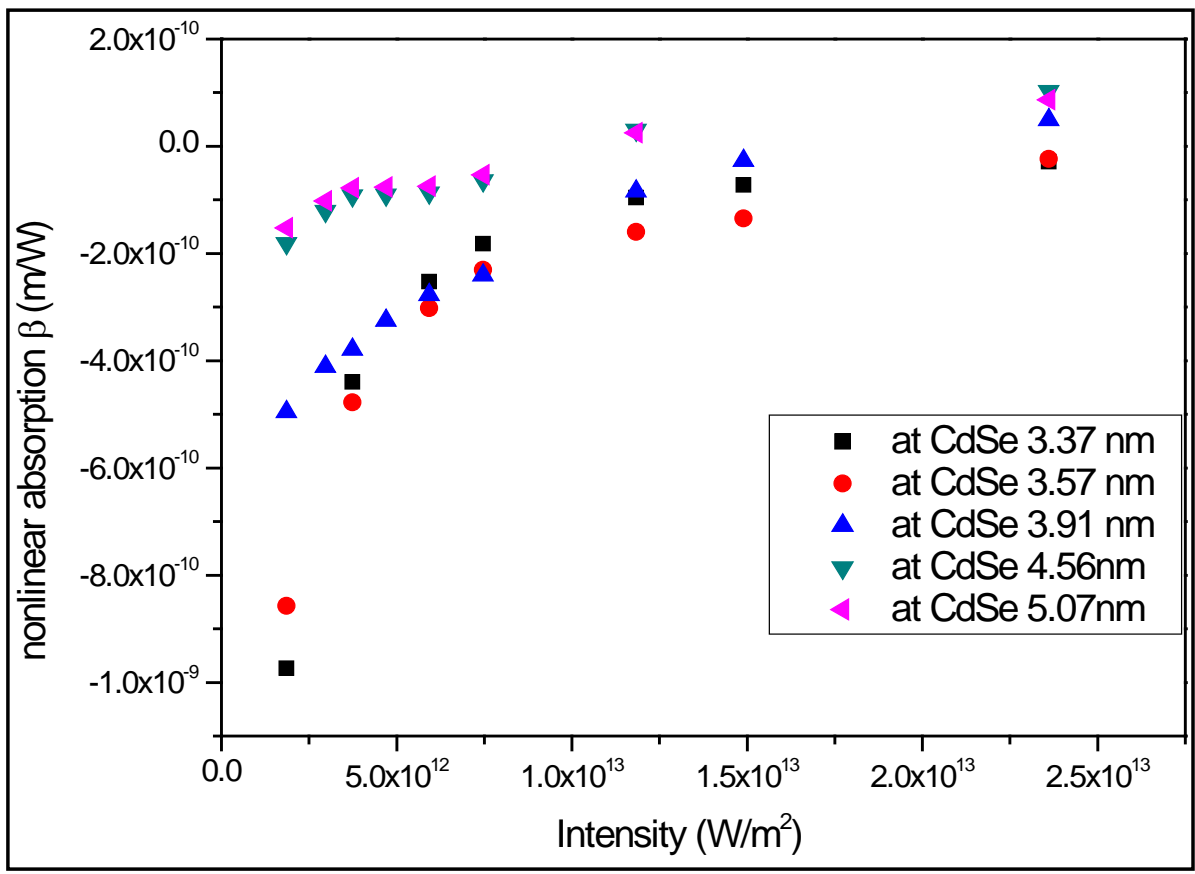

Figure 8. Dependance of the nonlinear aborption coefficient on Laser intensity for CdSe:Au of different size. 


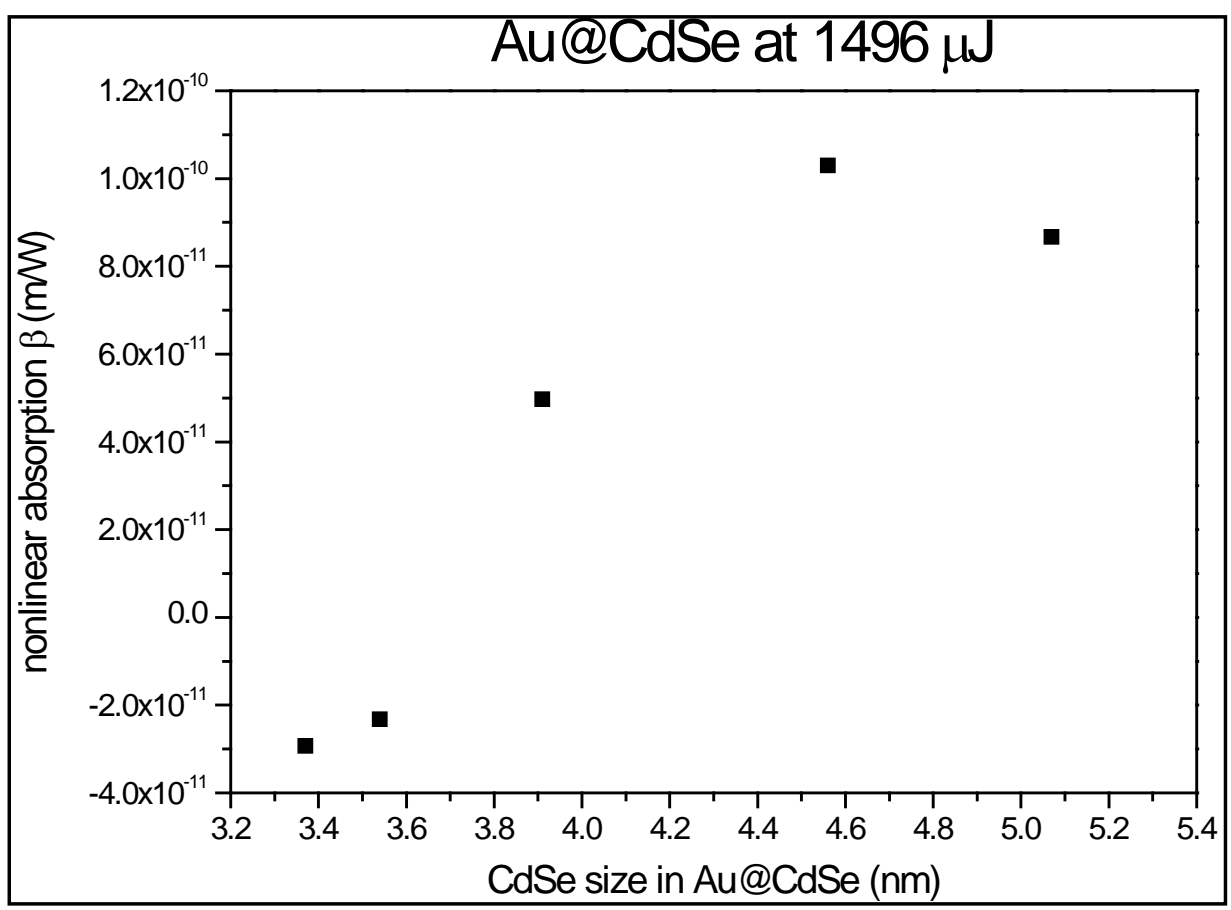

Figure 9. Dependance of the nonlinear absorption coefficient $\beta$ on composite size for CdSe:Au.

a pulse close to the absorption peak, they can't oscillate at the same frequency as that of the unexcited electrons. The excited electrons are free carriers possessing a whole spectrum of energies, causing the ground state absorption band to bleach or reduce in intensity which results increase in transmission in open aperture $\mathrm{z}$ scan. Apart of the excited electrons pumped to higher energy levels, causing excited state absorption, RSA will be observed at higher influences [15].

We studied the open aperture Z scan of toluene (solvent of the nanocomposites) to ensure that it hasn't nonlinear absorption effect using nanosecond laser, no change of the transmission was observed through focal point in open aperture $\mathrm{Z}$ scan of toluene solvent. This confirms that the nonlinear effect is from the prepared nanoparticles. Open aperture Z scan of toluene is shown in Figure 10.

The nonlinear absorption coefficients of hybrid CdSe:Au of CdSe size 3.91, 4.56 and $5.07 \mathrm{~nm}$ is $4.97 \times 10^{-11}$, $1.03 \times 10^{-10}$ and $8.67 \times 10^{-11} \mathrm{~m} / \mathrm{W}$ respectively. Comparing our results with that of ref. [16], because of the similar experimental conditions of nanosecond laser operating at $532 \mathrm{~nm}$, our measured values of nonlinear absorption of CdSe:Au are greater than the nonlinear absorption of CdSe QD [16] $\left(4.49 \times 10^{-11} \mathrm{~m} / \mathrm{W}\right.$ for size $\left.3.6 \mathrm{~nm}\right)$. We observed an enhancement of the nonlinear absorption coefficient of CdSe:Au at $5.07 \mathrm{~nm}$ more than two fold in comparison to CdSe [16]. This enhancement might be due to the existence of Au nanoparticles in the composite as well as the core-shell particles. Note that the measured value at energy $1496 \mu \mathrm{J}$ corresponding to intensity $\left(2.36 \times 10^{13} \mathrm{~W} / \mathrm{m}^{2}\right)$.

Elim et al. [17] illustrated that the nonlinear absorption coefficient of PbS-Au hybrid composites is attributed to the excitation of surface plasmon by energy transfer between PbS QDs and Au NPs through Auger recombination. The nonlinear absorption coefficient of saturable absorption in this composite is found to be dependent on excitation irradiance and it is determined to be $-2.9 \mathrm{~cm} / \mathrm{GW}$ at $78 \mathrm{GW} / \mathrm{cm}^{2}$. But the absolute non linear absorption of PbS QD-doped glasses was reported to be $0.75 \mathrm{~cm} / \mathrm{GW}$ by 15 ps laser pulses so the enhancement of nearly fourfold in comparison with that of pure PbS quantum dots QDs is observed.

Cesca et al. [18] investigated the nonlinear optical properties of Au-Ag nanoplanets close to their surface plasmon resonance by means of the single beam z-scan technique. Their results confirmed that both AuAg nanoplanets and AuAg nanoparticles exhibit large values of the nonlinear optical parameters with an opposite sign of the (intensity dependent) nonlinear absorption coefficient, demonstrating that at the highest investigated intensities (below the damage threshold) AuAg nanoplanets behaves as a saturable absorber, whereas AuAg nanoparticles exhibit optical limiting phenomena. They revealed strong local-field enhancement effects.

The optical limiting measurements are shown in Figure 11 for CdSe:Au, The differences in optical limiting 


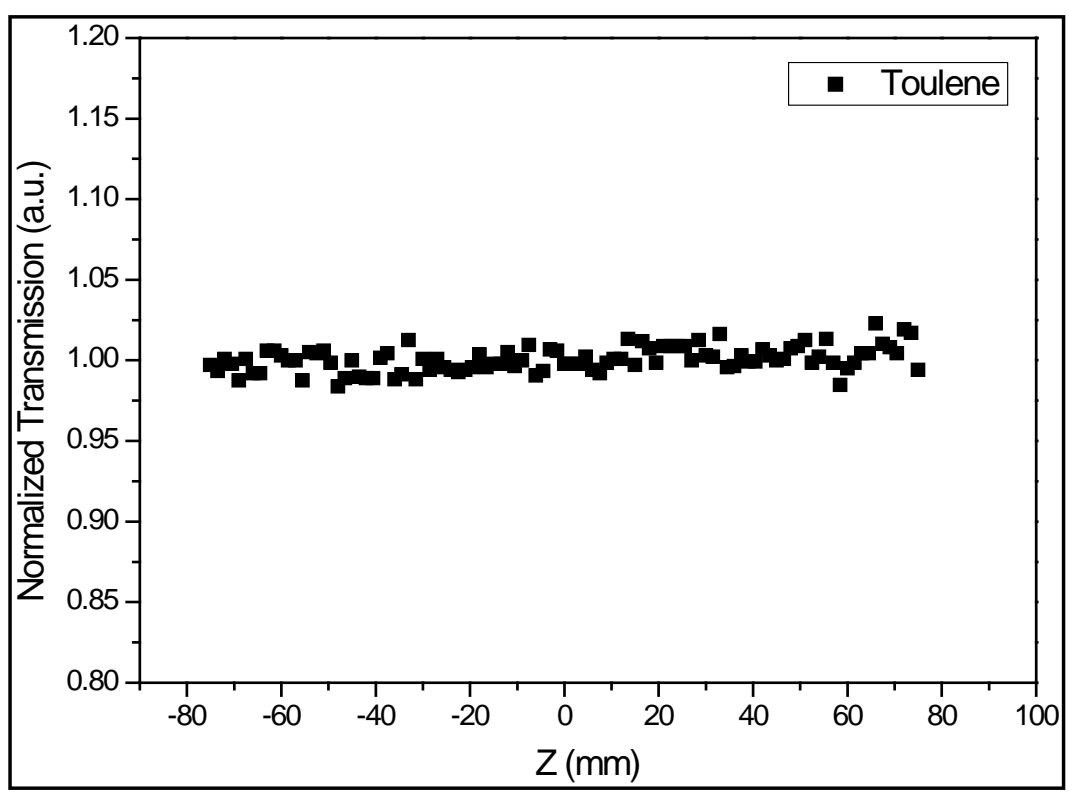

Figure 10. Open aperture $\mathrm{Z}$ scan of toluene which confirms that the nonlinear absorption using nanosecond laser is purely from hybrid nanocomposite.

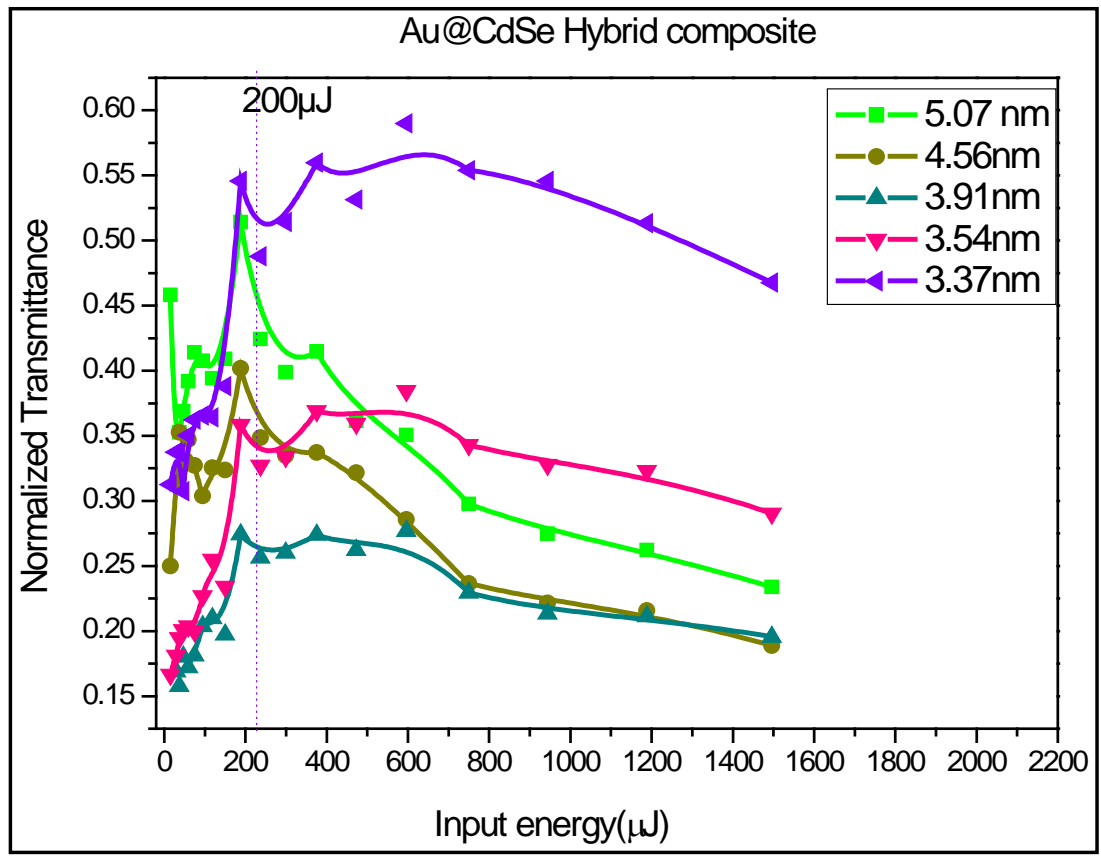

Figure 11. Optical limiting curve for hybrid CdSe:Au composite of different sizes at $532 \mathrm{~nm}$.

effects for those nanocomposites are observed for different sizes of the composites. Particles with larger size were found to have good optical limiting performance towards ns pulses. The enhanced performance of larger size of nanoparticles was ascribed to the increased free-carrier absorption for ns pulses. The presence of Au further improved the limiting performance of CdSe:Au.

\section{Conclusions}

For CdSe:Au, the combined SPR and excitonic peak position shifts from $538 \mathrm{~nm}$ to $613 \mathrm{~nm}$ as the particle sizes 
increase, at lower power densities, the nonlinear absorption is due to saturable absorption process. The nonlinear absorption coefficients were measured by open aperture Z-scan technique. The saturation absorption changes over to induced absorption when the intensity increased. Such changeover in the sign of the nonlinearity is related to the interplay of plasmon bleach and optical limiting mechanism. The absorption depth increases as the input intensity increases, and the SA decreases monotonically with increasing power density. This may be due to the cross section of the excited state is increased $(a t z=0)$ as the intensity increased so the reverse saturable become significant.

The prepared CdSe:Au composites have good optical limiting properties; these composites are promising for practical application. It can be used for various optical applications such as laser pulse compression and optical limiting for protecting optical sensors from high power laser pulses.

It can be concluded that the enhancement of optical nonlinearity for these hybrid composites mainly results from the contribution of gold metal nanoparticles and quantum confinement of CdSe. The large nonlinear absorption of the hybrid composite may allow them to be used as optical limiter in the opto-electronic field.

\section{Acknowledgements}

A. Salah thanks the National Institute of Laser Enhanced Science (NILES) for offering the required equipments for building Z scan Setup.

\section{References}

[1] Pan, H., Chen, W., Feng, Y.P., Jia, W. and Linb, J. (2006) Optical Limiting Properties of Metal Nanowires. Applied Physics Letters, 88, Article ID: 223106. http://dx.doi.org/10.1063/1.2208549

[2] Suchand Sandeep, C.S., Samal, A.K., Pradeep, T. and Philip, R. (2010) Optical Limiting Properties of Te and Ag $\mathrm{Ae}^{2}$ Nanowires. Chemical Physics Letters, 485, 326-330. http://dx.doi.org/10.1016/j.cplett.2009.12.065

[3] Huang, T., Hao, Z., Gong, H., Liu, Z., Xiao, S., Li, S., et al. (2008) Third-Order Nonlinear Optical Properties of a New Copper Coordination Compound: A Promising Candidate for All-Optical Switching. Chemical Physics Letters, 451, 213-217. http://dx.doi.org/10.1016/j.cplett.2007.12.001

[4] Takeda, Y., Plaksin, O.A., Lu, J. and Kishimoto, N. (2006) Optical Switching Performance of Metal Nanoparticles Fabricated by Negative Ion Implantation. Nuclear Instruments and Methods in Physics Research Section B: Beam Interactions with Materials and Atoms, 242, 194-197. http://dx.doi.org/10.1016/j.nimb.2005.08.016

[5] Muthukumar, V.S., Reppert, J., Sandeep, C.S.S., Krishnan, S.S.R., Podila, R., Kuthirummal, N., et al. (2010) Optical Limiting Properties of CdS Nanowires. Optics Communications, 283, 4104-4107. http://dx.doi.org/10.1016/j.optcom.2010.06.020

[6] Qu, S., Du, C., Song, Y., Wang, Y., Gao, Y., Liu, S., et al. (2002) Optical Nonlinearities and Optical Limiting Properties in Gold Nanoparticles Protected by Ligands. Chemical Physics Letters, 356, 403-408. http://dx.doi.org/10.1016/S0009-2614(02)00396-2

[7] Burda, C., Chen, X., Narayanan, R. and El-Sayed, M.A. (2005) Chemistry and Properties of Nanocrystals of Different Shapes. Chemical Reviews, 105, 1025-1102. http://dx.doi.org/10.1021/cr030063a

[8] Talapin, D.V., Lee, J.S., Kovalenko, M.V. and Shevchenko, E.V. (2010) Prospects of Colloidal Nanocrystals for Electronic and Optoelectronic Applications. Chemical Reviews, 110, 389-458. http://dx.doi.org/10.1021/cr900137k

[9] Shiek-Bahae, M., Said, A.A., Wei, T.-H. and Hagan, D.J. (1990) Sensistive Measurement of Optical Nonlinearities Using a Single Beam. IEEE Journal of Quantum Electronic, 26, 760-769. http://dx.doi.org/10.1109/3.53394

[10] Badr, Y., Salah, A., Elnaby, S.H., Sadek, A., Mohamed, M. and Azzouz, I. (2011) Analysis of Nonlinear Absorption of Au:CdSe Nanoshell. The 30th International Congress on Applications of Lasers \& Electro-Optics, Orlando, 23-27 October 2011, 179.

[11] AbouZeid, K.M., Mohamed, M.B. and El-Shall, M.S. (2011) Au-CdSe and Ag-CdSe Nanoflowers and Core-Shell Nanocrystals via “One-Pot” Heterogeneous Nucleation and Growth. Small, 7, 3299-3307. http://dx.doi.org/10.1002/smll.201100688

[12] Salah, A.M. (2012) Utilization of Nanopartilces for Nonlinear Optical Materials to Be Used in Photonic Devices. PhD, Cairo University, Cairo.

[13] Gerdova, I. and Haché, A. (2005) Third-Order Non-Linear Spectroscopy of CdSe and CdSe/ZnS Core Shell Quantum Dots. Optics Communications, 246, 205-212. http://dx.doi.org/10.1016/j.optcom.2004.10.068

[14] Zhang, J., Tang, Y., Lee, K. and Ouyang, M. (2010) Tailoring Light-Matter-Spin Interactions in Colloidal HeteroNanostructures. Nature, 466, 91-95. http://dx.doi.org/10.1038/nature09150 
[15] Anthony, J.K., Kim, H.C., Lee, H. W., Mahapatra, S.K., Lee, H.M., Kim, C.K., et al. (2008) Particle Size-Dependent Giant Nonlinear Absorption in Nanostructured Ni-Ti Alloys. Optics Express, 16, 11193-11202. http://dx.doi.org/10.1364/OE.16.011193

[16] Ma, S.M., Seo, J.T., Yang, Q., Battle, R., Brown, H., Lee, K., et al. (2006) Third-Order Nonlinear Susceptibility and Hyperpolarizability of CdSe Nanocrystals with Femtosecond Excitation. Journal of the Korean Physical Society, 48, 1379-1384.

[17] Elim, H.I., Ji, W., Yang, J. and Lee, J.Y. (2008) Intensity-Dependent Enhancement of Saturable Absorption in PbSAu4 Nanohybrid Composites: Evidence for Resonant Energy Transfer by Auger Recombination. Applied Physics Letters, 92, Article ID: 251106.

[18] Cesca, T., Calvelli, P., Battaglin, G., Mazzoldi, P. and Mattei, G. (2012) Local-Field Enhancement Effect on the Nonlinear Optical Response of Gold-Silver Nanoplanets. Optics Express, 20, 4537-4547. http://dx.doi.org/10.1364/OE.20.004537 